\title{
Estudiantes de ELE en el ámbito universitario de la Economía
}

\section{Análisis de estilos de aprendizaje e implicaciones didácticas}

\author{
Pilar Pérez Cañizares, Inmaculada Martínez Martínez ${ }^{2}$ \\ \& Johannes Schnitzer ${ }^{1}$ \\ ${ }^{1}$ Wirtschaftsuniversität Wien $\mid{ }^{2}$ Centro Internacional de Estudios \\ Superiores del Español - Comillas
}

Con el objetivo de crear en los estudiantes de español de la Universidad de Ciencias Económicas y Empresariales de Viena (Wirtschaftsuniversität Wien) una conciencia sobre sus preferencias y modos de aprender y promover así su autonomía, se realizó un estudio para establecer sus perfiles de aprendizaje. Para ello, se adaptó al alemán el cuestionario CHAEA (Alonso et al., 1994) y se diseñó una encuesta digital. Tras una fase de pilotaje, un panel de cinco expertos revisó la traducción al alemán. En la recogida de datos se consiguió una muestra altamente significativa: 198 de un total de 744 estudiantes $(26,6 \%)$. Los resultados muestran que entre los estudiantes de empresariales predominan básicamente dos perfiles de aprendizaje opuestos: la correlación de los estilos reflexivo-teórico y la correlación activo-pragmático. Esta dicotomía contrasta con los resultados de otras poblaciones y apunta a que los aprendientes podrían obtener beneficio de cualquier situación de aprendizaje, puesto que poseen estrategias de todos los estilos de aprendizaje.

Palabras clave: estilos de aprendizaje, cognitivismo, español como lengua extranjera, autonomía en el aprendizaje, diferencias individuales del aprendiz, español para fines específicos 


\section{Introducción}

Los intentos de mejorar la propia actuación docente se traducen, en muchos casos, en cambios en la metodología de enseñanza y en la creación de nuevos materiales con los que se pretende que, tanto profesores como estudiantes, se encuentren más cómodos y se enfrenten al aprendizaje con mayor motivación. Este afán por conseguir una docencia que se traduzca en un aprendizaje más eficiente y, en definitiva, más efectivo es solo posible si entendemos enseñanza y aprendizaje como ámbitos entre los que se establece una relación bidireccional y que deben estar en constante diálogo. Diagnosticar los estilos de aprendizaje de los estudiantes constituye una poderosa herramienta en manos del docente para adaptarse a las necesidades de sus alumnos y también a sus preferencias de aprendizaje (Tomlinson, 2014, p. 21).

La falta de consenso sobre la forma en que se adquiere una lengua extranjera (Consejo de Europa, 2002, p.138) debería llevar a los docentes, en tanto que facilitadores, a dar cabida en el aula a múltiples y diversas formas de aprender, que pueden eventualmente entrar en conflicto con la propia metodología docente (Dörnyei, 2005, p.155; Pinilla-Herrera y Cohen, 2019, p.43). La observación y constatación por parte del profesor de esta diversidad de comportamientos y preferencias en los alumnos a la hora de aprender puede tener dos consecuencias directas: en primer lugar, el docente puede intentar adaptar su metodología y las actividades de aula en función de las preferencias de los estudiantes (Peacock, 2001), o al menos gestionar las diferencias en el tiempo que estos necesitan para llevar a cabo las tareas (Yates, 2000); en segundo lugar, puede facilitarles el descubrimiento de nuevos modos de aprender con los que hasta entonces no habían tenido contacto o que no habían sabido aprovechar (style stretching, Ehrmann, 1996; Cohen, 2002, p.50). Esta intervención didáctica ha de ser cuidadosa y paulatina y en ningún caso debe suponer una transacción que suponga la eliminación de una preferencia ya existente para sustituirla por otra distinta a instancias de, por ejemplo, una metodología de enseñanza particular.

Es en este contexto y a partir de esta reflexión donde surge el presente trabajo. Con el objetivo de mejorar el proceso de enseñanza y aprendizaje en las clases de español de la Universidad de Ciencias Económicas y Empresariales de Viena (Wirtschaftsuniversität Wien) en el semestre de invierno de 2018 se llevó a cabo un estudio para conocer las formas de aprender de los estudiantes de español y establecer así en una primera fase sus perfiles de aprendizaje mayoritarios; a partir de un diagnóstico de estas características será posible hacer que los estudiantes universitarios de Empresariales (y también sus profesores) tomen conciencia de los modos preferidos a la hora de aprender y también de la posibilidad de aprovechar y desarrollar otros menos frecuentes, convirtiéndose en aprendientes cada 
vez más autónomos. Además, los datos podrán ser usados también para tomar decisiones respecto a la idoneidad de un tipo determinado de materiales didácticos y la adopción de una metodología de enseñanza concreta.

A continuación, se procederá a presentar el marco teórico y los principios en los que se basa este estudio. Posteriormente, se describirá la metodología utilizada y se analizarán los datos obtenidos a partir de la encuesta en que participaron 198 estudiantes universitarios de Empresariales (o carreras afines), que además estudian español para el ámbito profesional. Para finalizar, en los apartados de cierre se discutirán los resultados y se expondrán las conclusiones del estudio.

\section{Marco teórico}

\subsection{Los estilos de aprendizaje}

Se definen los estilos de aprendizaje como los rasgos cognitivos, afectivos y fisiológicos que sirven como indicadores, relativamente estables, de cómo los aprendices perciben, interactúan y responden al entorno de aprendizaje (Alonso et al., 1994). De las múltiples definiciones sobre los estilos de aprendizaje (entre otras, las de Kolb, 1976; Dunn y Dunn, 1978 y Keefe, 1979; una buena panorámica puede encontrarse en Coffield et al., 2004 y Renés y Martínez Geijo, 2015), este trabajo comparte la aportada por Honey y Mumford $(1986,1994)$, quienes los caracterizan basándose en la descripción de los comportamientos y las actitudes que hacen que el individuo tenga preferencia por un determinado tipo de aprendizaje.

Se toma como referencia el enfoque cognitivo que, interesado en el conocimiento, su adquisición y desarrollo, indaga en los procesos mentales que afectan al aprendizaje. Este se desarrolla en un proceso cíclico dividido en cuatro fases: tener una experiencia; racionalizar la experiencia; elaborar hipótesis y aplicar lo diseñado en correspondencia con los cuatro estilos, respectivamente: activo, reflexivo, teórico y pragmático (Figura 1).

Lo ideal, según Honey y Mumford $(1986,1994)$, es la existencia de un equilibrio entre los cuatro estilos, es decir, que los aprendientes sean capaces de experimentar, reflexionar, elaborar hipótesis y aplicar en proporciones iguales. En la práctica sabemos que no es así y que unos sujetos manifiestan diferentes preferencias a la hora de aprender. Los estilos de aprendizaje no son sino la interiorización, por parte de cada sujeto, de una etapa determinada del ciclo (Alonso et al., 1994, p. 69). El papel de la intervención didáctica sería, por tanto, el de ayudar a cubrir vacíos en una u otra etapa de ese proceso circular que es el aprendizaje. 


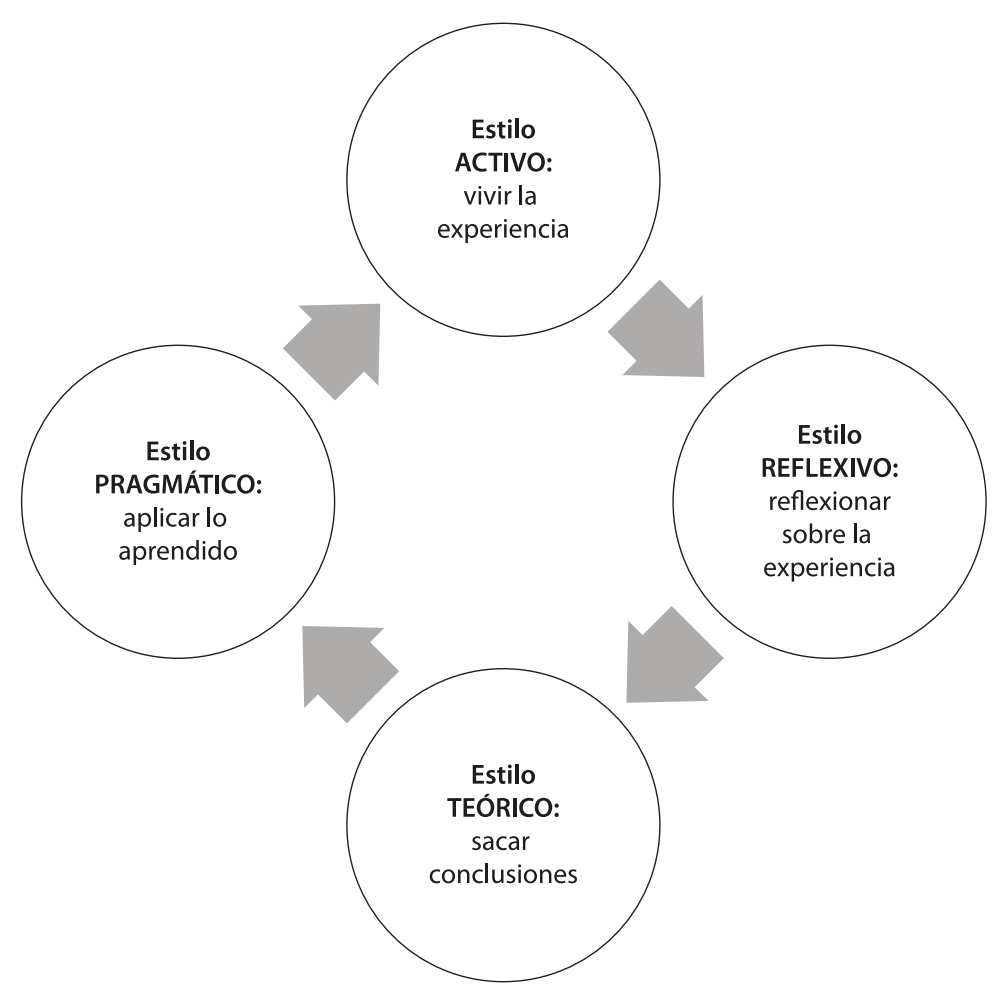

Figura 1. Los estilos de aprendizaje y las fases del enfoque cognitivo. (Fuente:

Elaboración propia)

De manera sintética, sus principales características son las siguientes (Martínez Martínez, 2004):

1. Activos: los estudiantes con preferencia alta o muy alta en este estilo se implican plenamente y sin prejuicios en nuevas experiencias. De mente abierta y nada escépticos, acometen con entusiasmo las tareas nuevas. Instalados en el presente -el aquí y el ahora- les encanta vivir nuevas experiencias. Se crecen ante los desafíos que suponen nuevas experiencias y se aburren con los plazos largos. Son personas a las que les gusta el grupo y centran alrededor todas las actividades. Suelen ser espontáneos, abiertos, creativos y arriesgados.

2. Reflexivos: a los estudiantes con preferencia alta o muy alta en este estilo les gusta considerar las experiencias y observarlas desde diferentes perspectivas. Recogen datos y los analizan con detenimiento antes de llegar a alguna conclusión. Son muy prudentes: prefieren tener en cuenta todas las alternativas posibles antes de llegar a alguna conclusión. Disfrutan al observar cómo 
actúan los demás y no intervienen hasta que se sienten seguros en la situación. Se caracterizan por ser ponderados, receptivos, exhaustivos y consecuentes.

3. Teóricos: los estudiantes con preferencia alta o muy alta en el estilo teórico adaptan e integran las observaciones que realizan dentro de teorías lógicas y complejas. Enfocan los problemas de forma vertical, escalonada, por etapas lógicas. Tienden a ser perfeccionistas e integran los hechos en teorías coherentes. Les gusta analizar y sintetizar. Son profundos en su sistema de pensamiento a la hora de establecer principios, teorías y modelos. Buscan la racionalidad y la objetividad huyendo de lo subjetivo y de lo ambiguo. Son lógicos, metódicos, críticos y perfeccionistas.

4. Pragmáticos: los estudiantes de preferencia alta o muy alta en el estilo pragmático se definen por buscar en todo momento la aplicación práctica de las ideas. Descubren el aspecto positivo de las nuevas ideas y aprovechan la primera oportunidad para experimentarlas. Les gusta actuar rápidamente y con seguridad con aquellas ideas y proyectos que les atraen. No les gustan las personas que teorizan: ellos buscan, ante todo, tomar una decisión o resolver un problema. Son eficaces, directos, prácticos y positivos.

De forma paralela al desarrollo de las distintas teorías de los estilos, se han confeccionado instrumentos para poder diagnosticar su tipología. Así, además de los ya citados, nos encontramos con autores que diseñaron instrumentos para su determinación. Algunos de los más utilizados son: Kolb (1976); Honey y Mumford (1986b); Dunn, Dunn y Price (1989); Vermunt (1994); Tait y Entwistle (1996) y Allinson y Hayes (1996). Además, existen también test o cuestionarios diseñados como instrumento de diagnóstico en particular en el campo de la adquisición de segundas lenguas, entre los que destacan los de Oxford (1993, 1999); Reid (1995); Cohen et al. (2001) y Ehrman y Leaver (2003).

Sin embargo, ni los estilos de aprendizaje como constructo, ni su parametrización, escapan a las críticas. Estas se alinean, sobre todo, en torno al protagonismo injustificado de los estilos en detrimento de otros factores que puedan condicionar el aprendizaje (Coffield et al., 2004, p.127), los problemas críticos con la medición al usar y forzar falsas dicotomías (An y Carr, 2017, p.411; Kirschner y van Merriënboer, 2013, p.16) o la ausencia de explicación de los mecanismos que causan las conductas que se pretenden medir (An y Carr, 2017, p.410). Además, otro problema adicional ligado a los estilos de aprendizaje es la cantidad de actividades lucrativas que se desarrollaron en cierto momento alrededor de los test, convirtiendo la realización de pruebas en todo un negocio (Pashler et al., 2008).

Ciertos autores incluso niegan absolutamente la existencia de los estilos de aprendizaje (Kirschner, 2017) y sobre todo, señalan la ausencia de evidencia científica que sustente la idea de que el aprendizaje será más eficiente si la metodolo- 
gía de enseñanza es acorde al estilo de aprendizaje (Pashler et al., 2008). En este sentido, siguiendo una afirmación de Honey (citado en Coffield et al., 2004, p. 83) opinamos que los instrumentos para recabar información sobre las distintas formas de aprender son, al menos, un punto de partida para mostrar a todos los actores implicados en el proceso que es posible aprender a aprender, y que hay un camino para lograr un aprendizaje autónomo. Este valor no lo niegan ni siquiera los autores más críticos con el constructo de los estilos de aprendizaje.

\subsection{Las diferencias individuales del aprendiz}

Los aprendientes difieren en maneras potencialmente infinitas, puesto que reflejan el completo rango de variables relacionadas con aspectos cognitivos, afectivos y sociales del ser humano (Ellis, 1994). El influjo que esta variación ejerza sobre el aprendiz afectará al aprendizaje de la L2 y se reflejará en el grado de progresión y en el nivel final de adquisición de la lengua (Martínez Martínez, 2004). Entre los factores que condicionan la variedad de los aprendices uno de los más estudiados es, sin duda, los estilos de aprendizaje.

El estudio sobre las diferencias individuales del aprendiz ha atraído una considerable atención en la investigación en adquisición de segundas lenguas y ha permitido numerosos avances. Junto a los estilos cognitivos, entre las dimensiones unánimemente reconocidas en la literatura sobre diferencias individuales en la enseñanza-aprendizaje de lenguas extranjeras se encuentran, entre otras, la edad, la aptitud, la motivación, las estrategias de aprendizaje y los resultados del aprendizaje. ${ }^{1}$ Uno de los objetivos de la investigación actual sobre diferencias individuales es, precisamente, identificar la naturaleza de todas sus interrelaciones (Ellis, 2004, p.547). Debe señalarse en este punto que son numerosos los estudios que en el nivel universitario analizan los estilos de aprendizaje en relación con estas variables (Freiberg et al., 2017). Así, los estilos han sido examinados en relación con variables psicológicas como las actitudes y la personalidad (Allueva-Torres y Bueno-García, 2011; Flores-Mejía et al., 2014); también existe bibliografía que respalda la relación entre los estilos de aprendizaje y variables sociodemográficas como la edad o el nivel educativo (Aguilar, 2010; Alducin-Ochoa y VázquezMartínez, 2017; Almigbal, 2015); finalmente, con respecto a las variables aca-

1. Los estilos cognitivos surgen en el seno de la psicología cognitiva e integran las distintas maneras en que las personas perciben la realidad de su entorno, procesan la información que obtienen y la almacenan en su memoria. La inserción del concepto de estilo cognitivo en un contexto de aprendizaje recibe el nombre de estilos de aprendizaje (cf. p.ej. Dörnyei y Skehan, 2003, p. 608 y ss.; Dörnyei, 2015, p. 106 y ss.). Siguiendo el ejemplo de otros estudios empíricos, ambos términos se utilizan en este trabajo como equivalentes. 
démicas, los estilos de aprendizaje se han estudiado en relación con la carrera cursada o con el rendimiento académico (véanse por ejemplo Acevedo-Pierart, 2011; Bangcola, 2016; Jahanbakhsh, 2012; Ventura y Moscoloni, 2015 y VillamizarAcevedo, 2012).

En este sentido, tanto para los docentes como los estudiantes, el interés por conocer los estilos de aprendizaje parece justificado: muchos son los autores que creen que una conciencia explícita de los aprendices sobre los procesos que subyacen al aprendizaje de la lengua puede conducirles a advertir tanto su singularidad como los rasgos que comparten con otros aprendices. En el caso de los profesores, y en su papel de facilitadores, es esencial que estos consideren la importancia de la variación en los estudiantes -mediante el estilo cognitivo, por ejemplo-, la traten abiertamente en clase y proporcionen a sus estudiantes guías y directrices sobre cómo gestionar dicha variedad (MacIntyre et al., 2016). Frente a otras dimensiones individuales, como la edad o la aptitud, con la que los estudiantes poco o nada pueden hacer, los estilos de aprendizaje son modificables (Ellis, 1994).

Como docentes, conducir a los estudiantes a que regulen y controlen su propio aprendizaje es facilitarles las herramientas para que dicho aprendizaje sea más sencillo y efectivo y para que ellos mismos se conviertan en más autónomos y activos en el proceso (Dörnyei y Skehan, 2003; Cohen, 2007).

Las fases de este ejercicio de sensibilización que los profesores pueden llevar a cabo con los aprendices y este aspecto de su variación individual parecen claras y se muestran en la Figura 2:

1. Comenzar con un cuestionario sobre estilos de aprendizaje como el que aquí se presenta.

2. Explicar las diferencias individuales que existen en las preferencias de aprendizaje y favorecer una introspección al respecto.

3. Buscar el diagnóstico personal de cada uno y hacerles conscientes a todos de sus fortalezas y de los aspectos por mejorar.

4. Iniciar en el aula procesos de autorregulación que tomen como base el diagnóstico conseguido.

El docente facilitará una serie de herramientas o estrategias para que el estudiante reflexione sobre su propio proceso de aprendizaje (autocognición). Estas le permitirán, por un lado, reducir el impacto de factores improductivos o perjudiciales (Young, 1991). Por otro lado, les ayudarán en su camino a la autonomía y, de ahí, a una mayor eficacia en el aprendizaje (MacIntyre et al., 2016). Parece existir un efecto positivo en el rendimiento cuando los estudiantes se enfrentan a algo nuevo mientras prueban nuevos estilos de aprendizaje y cuando los profesores les permiten sacar el máximo rendimiento a la variedad y diversidad inserta en ellos 


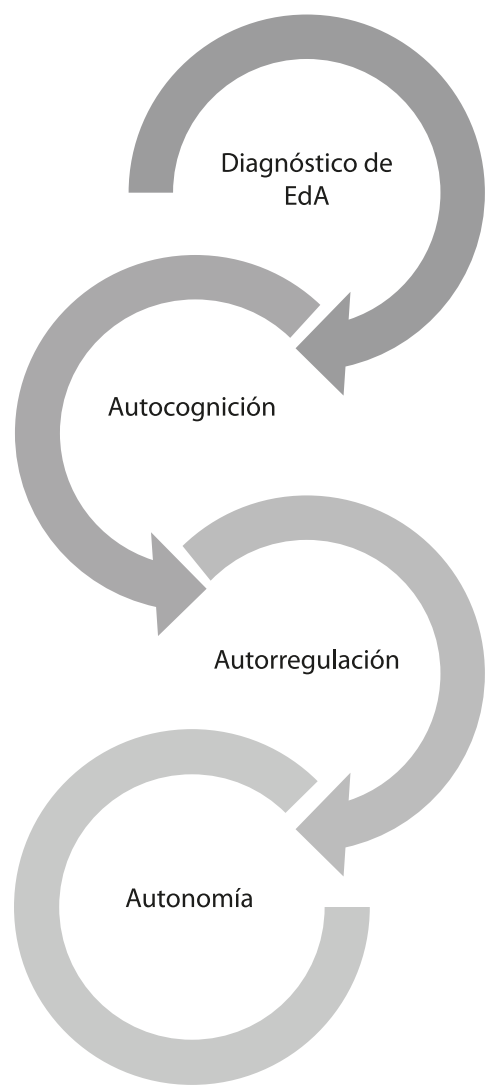

Figura 2. Los estilos de aprendizaje y su tratamiento en el aula. (Fuente: Elaboración propia)

y les ayudan a encontrar maneras de hacerlo por sí mismos dentro y fuera de clase (Gregersen y MacIntyre, 2014).

\section{Metodología y presupuestos básicos}

\subsection{Objetivos y población de referencia}

Como hemos mencionado arriba, el objetivo final de este proyecto es el de dar una mejor respuesta a la diversidad de estilos de aprendizaje de los estudiantes de español de la Universidad de Ciencias Económicas y Empresariales de Viena (Wirtschaftsuniversität Wien), tanto respecto a los materiales didácticos utilizados como a la práctica docente en general. En concreto, esta mejora en el intercambio docencia / aprendizaje se traduce, por una parte, en sensibilizar a los estudiantes 
sobre la posibilidad de ampliar su propio perfil de aprendizaje y, por otra, en mejorarlo. El objetivo es no solo adaptarnos en la medida de lo posible a sus preferencias de aprendizaje y sentar así las bases para una metodología docente con la que se encuentren más cómodos, sino también mostrarles que existen otros modos de aprender distintos a los que ellos normalmente usan. Se pretende, pues, acompañarlos en el descubrimiento de estas posibilidades que aún les son ajenas, y facilitarles así de manera paulatina el aprendizaje también en entornos a los que están menos habituados o en los que se encuentran menos cómodos.

En definitiva, se trata de aprovechar las circunstancias en las que mejor aprenden, pero a la vez familiarizarlos con otras para así ampliar gradualmente el número de situaciones favorables para el aprendizaje (Gregersen y MacIntyre, 2014, p.182; Dörnyei, 2015, p.137). El hecho de que los estudiantes, a lo largo de su proceso de aprendizaje, vayan conociendo cada vez más posibilidades de aprender tendrá como consecuencia un aumento en la autonomía de aprendizaje, puesto que serán capaces de aprender en un mayor número de situaciones.

Para ello, como paso previo, procedimos a realizar un diagnóstico sobre los estilos de aprendizaje de los estudiantes de español de nuestra institución, la Universidad de Ciencias Económicas y Empresariales de Viena (Wirtschaftsuniversität Wien). La gran mayoría de estudiantes que cursan español en esta universidad lo hacen en el marco de cuatro asignaturas obligatorias dentro del plan de estudios del grado Ciencias Empresariales Internacionales. Dentro de esta carrera, los estudiantes deben aprender dos idiomas que pueden elegir de entre inglés, español, francés, italiano y ruso. Además, tienen la opción de cursar dos asignaturas optativas de español general (nivel A1/ A2 y nivel A2+/ B1) que en principio están pensadas para alcanzar el nivel de las cuatro asignaturas semestrales obligatorias que prevé el programa. Las asignaturas obligatorias las constituyen cuatro cursos de nivel $\mathrm{B}_{1}+$ hasta $\mathrm{B}_{2}+$ con contenidos de español para fines profesionales, español del ámbito empresarial y español comercial. Estas mismas asignaturas pueden cursarse como optativas dentro de otros estudios de grado como Ciencias Empresariales, Derecho Económico o Informática Económica, de modo que la muestra puede incluir a estudiantes que cursan grados distintos, pero todos ellos con cierta orientación a las Ciencias Empresariales y/o Económicas. También en el caso de estudios de máster es posible elegir una asignatura de Español de los Negocios. En los últimos años, el profesorado ha realizado un esfuerzo para que los contenidos de esos cursos presenten un equilibrio entre actividades comunicativas escritas y orales, tanto en la práctica, como en la evaluación.

El alumnado, en general, está formado por estudiantes de entre 19 y 25 años procedentes en parte de Austria, pero también de Alemania y de otros países vecinos como Hungría, Eslovaquia, Serbia, Rumanía o Croacia. Otro de los objetivos del estudio consiste, pues, en establecer si existen diferencias respecto a los estilos de aprendizaje basadas en estas variables socioacadémicas, es decir, si existen 
diferencias en los estilos de aprendizaje, por ejemplo, entre hombres y mujeres, o entre los estudiantes que tienen el alemán como lengua materna y aquellos que no.

\subsection{Herramienta y diseño de la encuesta}

Para el diagnóstico del perfil de aprendizaje de los estudiantes, se utilizó el cuestionario Honey-Alonso de estilos de aprendizaje (CHAEA) (Alonso et al., 1994) como instrumento de obtención de datos. Este test consta de 80 ítems de elección dicotómica (sí / no), de los cuales 20 corresponden a cada uno de los cuatro estilos de aprendizaje caracterizados en el apartado anterior y se presentan en un orden aleatorio. El CHAEA se originó a mediados de los años 9o del siglo pasado como una adaptación del cuestionario Learning Styles Questionnaire (LSQ) (Honey y Mumford, 1986b) al contexto de la educación universitaria española (Alonso et al., 1994). Ambos cuestionarios, al igual que otros instrumentos comparables, han recibido críticas acerca de su confiabilidad y validez. Respecto al LSQ, Coffield et al. (2004, p. 82) resumen los resultados de investigaciones previas para concluir que la fiabilidad de la consistencia interna del LSQ es solo moderada y que su validez para predecir el rendimiento académico es limitada, aunque sí presenta fiabilidad test-retest. Una actualización reciente del trabajo de Coffield et al. (2004) la constituye el estudio de Álvarez-Montero et al. (2018), que revisa la fiabilidad y validez de varios instrumentos de recogida de datos sobre estilos de aprendizaje y llega a conclusiones similares a las de sus predecesores; en concreto, en el caso del LSQ, se examinan cuatro investigaciones previas que coinciden en la incapacidad del cuestionario para predecir rendimiento. Respecto al CHAEA, el trabajo de Escurra Mayaute (2011) llega a conclusiones contradictorias respecto a su fiabilidad, dependiendo del modelo matemático con el que se evalúe. El panorama de la investigación, pues, reúne un coro de voces mayoritariamente críticas respecto a la fiabilidad de los instrumentos, pero demasiado cautas a la hora de ofrecer alternativas o dar directrices sobre cómo enfocar metodológicamente posibles estudios sobre estilos de aprendizaje (Coffield et al. 2004; Álvarez-Montero et al. 2018).

En este contexto, la elección de la herramienta viene justificada por la intención de obtener datos comparables con otros estudios realizados anteriormente (Alonso et al., 1994; Martínez Martínez, 2004). Además, algunos trabajos (Van Zwanenberg et al., 200o) sugieren que el LSQ, adaptado por Alonso et al., (1994) al contexto de la educación universitaria española en forma del CHAEA, es más fiable a la hora de obtener resultados sobre población universitaria que, por ejemplo, el Index of Learning Styles (ILS), desarrollado por Felder y Silverman (1988).

Una vez seleccionada la herramienta para la obtención de datos, se procedió en primer lugar a su traducción al alemán. No nos consta la existencia de nin- 
guna otra versión alemana del cuestionario que haya sido utilizada con anterioridad en un estudio de este tipo. Para ello, un panel de expertos realizó una traducción de los 80 ítems del cuestionario. Para tratar de solventar las dificultades de interpretación presentadas por algunos de los ítems, se recurrió a la versión original en inglés creada por Honey y Mumford (1986b). La versión alemana fue, además, revisada por pares en una fase posterior y modificada ligeramente tras la fase de pilotaje.

El siguiente paso consistió en elaborar una encuesta que incluyera el cuestionario y que, además, recabara datos personales y académicos de los participantes. Para ello, se utilizó la aplicación de software LimeSurvey, una herramienta para la realización de encuestas en línea en la que, aparte del cuestionario, se recopilaron algunos datos socioacadémicos, que fueron usados como variables independientes en las pruebas estadísticas posteriores. ${ }^{2}$ La encuesta fue diseñada para que los participantes solo tuvieran que seleccionar la opción que les correspondía en cada una de las preguntas. Se limitaba así todo lo posible la introducción de campos libres.

Tabla 1. Datos socioacadémicos recabados en la encuesta

\begin{tabular}{ll}
\hline Variable & Comentario \\
\hline Sexo: & $\begin{array}{l}\text { Se presentaban las siguientes opciones: mujer, hombre y otro (con } \\
\text { posibilidad de completar en campo libre) }\end{array}$ \\
$\begin{array}{l}\text { Año de nacimiento: } \\
\text { Lengua materna: }\end{array}$ & $\begin{array}{l}\text { Para seleccionar en un menú desplegable } \\
\text { Pregunta inicial dicotómica sobre el alemán como lengua materna (sí/ } \\
\text { no); en caso de que la respuesta sea negativa, opción de elegir entre } \\
\text { otras lenguas frecuentes, o de escribirla }\end{array}$ \\
$\begin{array}{l}\text { Examen final de la } \\
\text { enseñanza media } \\
\text { (Matura): }\end{array}$ & $\begin{array}{l}\text { Respuesta dicotómica (sí / no) } \\
\text { Conocimiento de }\end{array}$ \\
$\begin{array}{l}\text { Tabliomas y nivel: } \\
\text { que los participantes piensan tener. Aparecen los idiomas inglés, francés, } \\
\text { italiano, español, ruso, polaco, chino, japonés, árabe y es posible añadir } \\
\text { manualmente otros adicionales. }\end{array}$ \\
$\begin{array}{l}\text { Estudios en curso de } \\
\text { realización: }\end{array}$ & $\begin{array}{l}\text { Opciones: los estudios de grado y máster ofertados en la institución, } \\
\text { todos ellos dentro del campo las Ciencias Empresariales y/o Económicas }\end{array}$ \\
\hline
\end{tabular}

La recogida de los datos anteriores viene condicionada por las hipótesis de las que parte este trabajo, en concreto, si alguna de las variables anteriores puede

2. https://www.limesurvey.org/es/ 
implicar alguna diferencia en el perfil de aprendizaje de la población estudiada. En definitiva, se trata de averiguar, por ejemplo, si el conocimiento de un mayor número de idiomas implica que las personas que los hablan tienen unas preferencias respecto a su propio aprendizaje distintas a las de individuos que no las hablan; o si el haber cursado la enseñanza obligatoria preuniversitaria en un sistema educativo concreto, como, por ejemplo, el de Austria, condiciona los estilos de aprendizaje de algún modo que haga que los individuos que hayan estado inmersos en él se diferencien de aquellos que no lo hayan estado. La selección de las variables, por tanto, obedece en primer lugar a la posibilidad de formular hipótesis razonables y comprobables a partir de ellas. Otro factor importante que influye en la selección de variables es hasta qué punto la población examinada está dispuesta a proporcionar cierto tipo de información. En este sentido, por ejemplo, en este estudio se renunció a formular hipótesis sobre correlaciones entre estilos de aprendizaje y rendimiento académico, puesto que se trata de un dato que los participantes en el estudio quizá no habrían deseado compartir y habría repercutido negativamente en el tamaño de la muestra.

La encuesta se presentó como colaboración voluntaria para un proyecto de investigación, en la que los participantes obtendrían además información sobre sus propios estilos de aprendizaje. Sin embargo, no se proporcionó ningún tipo de explicación previa sobre el modelo teórico en el que nos basamos (ni siquiera la distinción entre los cuatro estilos de aprendizaje), para no predisponer en modo alguno a los participantes a la hora de aportar sus respuestas. La herramienta fue diseñada de modo que, inmediatamente después de rellenar el cuestionario, se calcula la puntuación obtenida en cada uno de los estilos y se da la posibilidad de consultar unas breves recomendaciones para mejorar cada uno de ellos, si el participante así lo desea.

A diferencia de otros estudios similares, una vez rellenado el cuestionario y antes de proporcionar la puntuación obtenida, se presentó una breve caracterización sobre los cuatro estilos de aprendizaje y se pidió a los participantes que se otorgaran a sí mismos una puntuación entre o y 20 para cada uno de ellos. Esta breve fase de autoevaluación se introdujo para provocar una reflexión sobre las propias preferencias de aprendizaje.

\subsection{Pilotaje de la encuesta}

La fase de pilotaje de la herramienta se llevó a cabo entre el 26 de septiembre y el 16 de octubre de 2018. Para ello se pidió la colaboración de los estudiantes de un curso intensivo de español que tuvo lugar durante esas semanas. En total, 20 estudiantes rellenaron la encuesta y dieron retroalimentación sobre ella en breves entrevistas personales. A partir de los comentarios de estos estudiantes, un panel 
de cinco expertos revisó la traducción al alemán del cuestionario. A raíz de ello, se procedió a corregir algunos errores técnicos de la encuesta en línea (p.ej. posibilidad de marcar varias casillas en preguntas para las que se prevé una única respuesta) y se modificó la traducción de uno de los ítems del cuestionario (pregunta 68). Puesto que se realizaron algunos cambios, los datos de los participantes en la fase de pilotaje no se incluyeron en los del estudio.

\subsection{Recogida de datos}

Una vez modificada y corregida la encuesta, esta se dio a conocer en las clases presenciales de español de la institución a través de una presentación del proyecto de investigación para establecer los estilos de aprendizaje del alumnado de español. Todos los alumnos participantes en cursos de español durante el semestre de invierno de 2018 recibieron además un correo electrónico a través de la plataforma de aprendizaje en el que se les invitaba a colaborar en el estudio. La encuesta estuvo activa entre el 16 de octubre y el 13 de diciembre de 2018.

\section{Análisis de los datos}

A continuación se presentan los resultados obtenidos mediante la aplicación de la encuesta. La muestra obtenida fue de 198 cuestionarios, es decir, algo más de una cuarta parte de la población de referencia (744 estudiantes de español en la Universidad de Ciencias Económicas y Empresariales de Viena (Wirtschaftsuniversität Wien) en el semestre de invierno de 2018), cuyas características resumimos en la Tabla 2.

Una vez determinados los estilos de aprendizaje a partir de la traducción al alemán del cuestionario CHAEA, estos se contrastaron con los valores que los participantes se atribuyeron en la autoevaluación para cada uno de los estilos y, a continuación, con las seis variables socioacadémicas recabadas en la encuesta. Posteriormente, se procedió a establecer correlaciones entre estilos para poder así determinar posibles perfiles de aprendizaje. En la Tabla 3 presentamos la media de los resultados obtenidos en cada uno de los estilos de aprendizaje mediante la aplicación de la versión alemana del cuestionario CHAEA. En ella se observa que las medias más elevadas corresponden a los estilos reflexivo y teórico y que la media obtenida en el estilo activo es, con diferencia, la más baja.

Las medias obtenidas en el cuestionario CHAEA en cada uno de los estilos de aprendizaje contrastan con las medias de las puntuaciones que los participantes se otorgaron a sí mismos en la autoevaluación. Estas medias se presentan a continuación, en la Tabla 4. 
Tabla 2. Distribución de las variables socioacadémicas

\begin{tabular}{|c|c|c|c|c|c|}
\hline \multirow{2}{*}{\multicolumn{2}{|c|}{$\begin{array}{l}\text { Variable } \\
\text { Sexo: }\end{array}$}} & \multirow{2}{*}{$\begin{array}{l}\text { Resultados } \\
131 \text { mujeres } \\
(66,2 \%)\end{array}$} & & \multicolumn{2}{|l|}{ Comentario } \\
\hline & & & $\begin{array}{l}67 \text { hombres } \\
(33,8 \%)\end{array}$ & & \\
\hline \multicolumn{2}{|l|}{ Edad: } & \multicolumn{4}{|c|}{$\begin{array}{l}\text { más del 90\% de los } \\
\text { participantes entre } 19 \text { y } 24 \\
\text { años }\end{array}$} \\
\hline \multicolumn{2}{|c|}{ Lengua materna: } & $\begin{array}{l}169 \text { alemán } \\
(85,3 \%)\end{array}$ & $\begin{array}{l}29 \text { no } \\
\text { alemán } \\
(14,6 \%)\end{array}$ & \multicolumn{2}{|c|}{$\begin{array}{l}\text { Las otras lenguas maternas más } \\
\text { frecuentes (aparte del alemán) } \\
\text { fueron el húngaro (8) y el serbio } \\
\text { (6). }\end{array}$} \\
\hline \multicolumn{2}{|c|}{$\begin{array}{l}\text { Examen final de la enseñanza } \\
\text { media (Matura): }\end{array}$} & $\begin{array}{l}173 \text { sí } \\
(87,3 \%)\end{array}$ & $\begin{array}{l}25 \text { no } \\
(12,6 \%)\end{array}$ & & \\
\hline \multicolumn{2}{|c|}{$\begin{array}{l}\text { Conocimiento de } 3 \text { o más } \\
\text { lenguas extranjeras } \\
\text { (independientemente del nivel): }\end{array}$} & $\begin{array}{l}112 \text { sí } \\
(56,6 \%)\end{array}$ & $\begin{array}{l}86 \text { no } \\
(46,4 \%)\end{array}$ & \multicolumn{2}{|c|}{$\begin{array}{l}\text { El establecimiento del rango de } 3 \\
\text { o más lenguas extranjeras se } \\
\text { realizó a posteriori, a partir de } \\
\text { los datos. }\end{array}$} \\
\hline \multicolumn{6}{|c|}{ Estudios en curso de realización } \\
\hline $\begin{array}{l}\text { Empresariales } \\
48(24 \%)\end{array}$ & $\begin{array}{l}\text { Empresariales } \\
\text { Internacionales } \\
123(62 \%)^{\star}\end{array}$ & $\begin{array}{l}\text { Económicas } \\
5(3 \%)\end{array}$ & $\begin{array}{l}\text { Informática } \\
\text { Económica } \\
4(2 \%)\end{array}$ & $\begin{array}{l}\text { Economía y } \\
\text { Derecho } \\
8(4 \%)\end{array}$ & $\begin{array}{l}\text { Programa de } \\
\text { máster } \\
10(5 \%)\end{array}$ \\
\hline
\end{tabular}

* Incluye 10 estudiantes de esta carrera en combinación con otra.

Tabla 3. Medias de las puntuaciones obtenidas en cuestionario CHAEA en cada uno de los estilos de aprendizaje

\begin{tabular}{lcccc}
\hline$N=198$ & $\begin{array}{c}\text { Resultado estilo } \\
\text { activo }\end{array}$ & $\begin{array}{c}\text { Resultado estilo } \\
\text { reflexivo }\end{array}$ & $\begin{array}{c}\text { Resultado estilo } \\
\text { teórico }\end{array}$ & $\begin{array}{c}\text { Resultado estilo } \\
\text { pragmático }\end{array}$ \\
\hline Media & 10,72 & 14,20 & 13,16 & 12,37 \\
S. D. & 3,399 & 2,716 & 2,820 & 3,158 \\
\hline
\end{tabular}

* Standard Deviation o desviación estándar.

Los participantes en el estudio, en promedio, se otorgaron una puntuación en los estilos de aprendizaje reflexivo, teórico y pragmático muy similar a la que de hecho obtuvieron en el cuestionario CHAEA. Únicamente respecto al estilo activo hay una gran diferencia entre ambos valores, lo que apunta a que, en general, los participantes creen ser bastante más activos de lo que en realidad son. 
Tabla 4. Medias de las puntuaciones que los participantes se otorgaron en cada uno de los estilos de aprendizaje en la autoevaluación

\begin{tabular}{lcccc}
\hline$N=\mathbf{1 9 8}$ & $\begin{array}{c}\text { Autoevaluación } \\
\text { estilo activo }\end{array}$ & $\begin{array}{c}\text { Autoevaluación } \\
\text { estilo reflexivo }\end{array}$ & $\begin{array}{c}\text { Autoevaluación } \\
\text { estilo teórico }\end{array}$ & $\begin{array}{c}\text { Autoevaluación } \\
\text { estilo pragmático }\end{array}$ \\
\hline Media & 13,06 & 13,99 & 12,37 & 11,80 \\
S. D. & 4,211 & 4,231 & 4,448 & 4,876 \\
\hline
\end{tabular}

La comparación de los resultados del cuestionario y los de la autoevaluación se puede ver en la Figura 3.

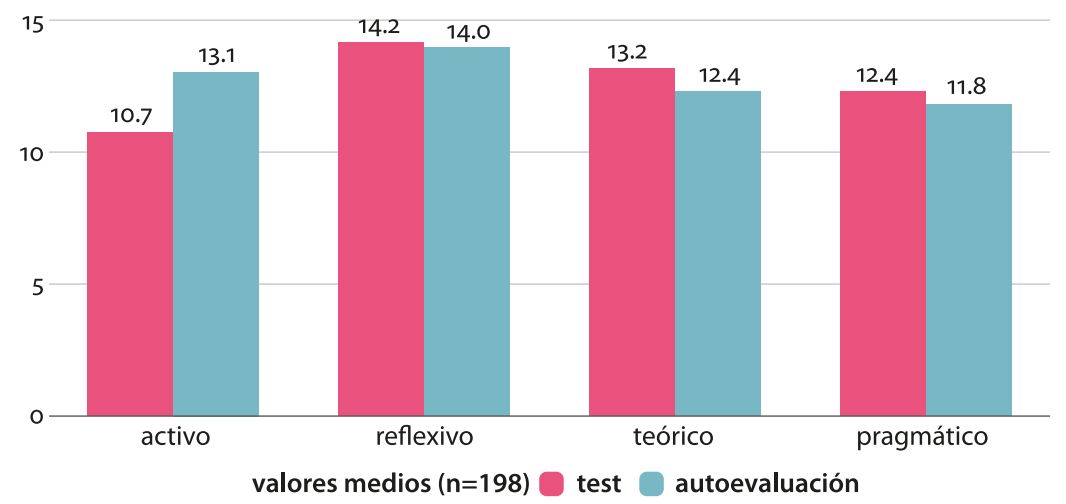

Figura 3. Comparación de los resultados del cuestionario y los resultados de la autoevaluación. (Fuente: Elaboración propia)

\subsection{Baremo general abreviado}

A la hora de interpretar las puntuaciones obtenidas individualmente en cada uno de los estilos de aprendizaje, debemos considerar que se trata de valores relativos que deben calibrarse en relación con los resultados del resto de individuos que conforman la muestra. Siguiendo estudios anteriores (Alonso et al., 1994, p.112 y ss.; Martínez Martínez, 2004), hemos establecido un baremo para facilitar la interpretación de las puntuaciones individuales: una puntuación obtenida en uno de los estilos de aprendizaje que esté cercana a la media se traducirá en una preferencia moderada por ese estilo de aprendizaje. Las preferencias alta y muy alta indicarán que esa puntuación se sitúa entre el $20 \%$ o el $10 \%$, respectivamente, de puntuaciones más altas obtenidas por los participantes. A su vez, una preferencia baja o muy baja significa que la cifra obtenida sitúa a esa persona entre el 10\% o el $20 \%$ respectivamente de personas con menor puntuación en ese estilo. Las dife- 
rencias a la hora de calibrar cada uno de los estilos de aprendizaje se pueden resumir en la tabla siguiente:

Tabla 5. Preferencias en estilos de aprendizaje

\begin{tabular}{|c|c|c|c|c|c|}
\hline$N=198$ & $\begin{array}{l}\quad 10 \% \\
\text { Preferencia } \\
\text { MUY BAJA }\end{array}$ & $\begin{array}{c}20 \% \\
\text { Preferencia } \\
\text { BAJA }\end{array}$ & $\begin{array}{c}40 \% \\
\text { Preferencia } \\
\text { MODERADA }\end{array}$ & $\begin{array}{c}20 \% \\
\text { Preferencia } \\
\text { ALTA }\end{array}$ & $\begin{array}{c}\quad 10 \% \\
\text { Preferencia } \\
\text { MUY ALTA }\end{array}$ \\
\hline activo & $0-6$ & $7-9$ & $\begin{array}{c}\text { 10-12 } \\
\text { (Media: } \\
10,72)\end{array}$ & $13-14$ & $15-20$ \\
\hline reflexivo & $0-10$ & $11-12$ & $\begin{array}{c}13-15 \\
\text { (Media: } \\
14,20 \text { ) }\end{array}$ & $16-17$ & $18-20$ \\
\hline teórico & $0-9$ & $10-11$ & $\begin{array}{c}\text { 12-14 } \\
\text { (Media: } \\
13,16)\end{array}$ & $15-16$ & $17-20$ \\
\hline pragmático & $0-8$ & $9-10$ & $\begin{array}{c}\text { 11-14 } \\
\text { (Media: } \\
12,37)\end{array}$ & $15-16$ & $17-20$ \\
\hline
\end{tabular}

4.2 Correlaciones entre los estilos de aprendizaje

Una vez calculadas las medias en cada uno de los estilos de aprendizaje y aplicado un baremo para interpretar el grado de preferencia en cada uno de ellos según la puntuación obtenida individualmente, es posible establecer también correlaciones (positivas o negativas) entre los distintos estilos, lo que permitirá trazar una visión de conjunto de posibles perfiles de aprendizaje en tanto que combinaciones de los cuatro estilos. El coeficiente de correlación de Pearson ofrece las siguientes relaciones entre los estilos de aprendizaje de nuestros estudiantes:

Tabla 6. Matriz de correlación de Pearson

\begin{tabular}{lcccc}
\hline & Activo & Reflexivo & Teórico & Pragmático \\
\hline activo & 1 & & & \\
reflexivo & $-0,344$ & 1 & & \\
teórico & $-0,317$ & 0,4 & 1 & 1 \\
pragmático & 0,356 & $-0,089$ & 0,182 & 1 \\
\hline
\end{tabular}


La Tabla 6 muestra más oscuras las correlaciones positivas, es decir, los casos en los que una preferencia alta por un estilo de aprendizaje en particular va ligada a un valor también alto en otro estilo. En nuestro caso concreto, la correlación más alta es la de los estilos reflexivo y teórico, seguida de cerca por la de los estilos activo y pragmático. Con un coeficiente bastante más bajo, la tercera correlación que se establece a partir de nuestros datos es la de los estilos teórico y pragmático. La obtención de un índice de correlación negativo es también ilustrativa y apunta a que los dos estilos en cuestión (en nuestro caso, por ejemplo, el estilo activo y el reflexivo) son incompatibles, es decir, una preferencia alta por uno de estos estilos viene ligada a una preferencia baja en el otro.

\subsection{Influencia de los datos socioacadémicos en los estilos de aprendizaje}

Como ya hemos mencionado arriba, en la encuesta se incluyeron algunas preguntas para recabar datos como edad, sexo, lengua materna, escolarización en el sistema educativo austriaco (realización de la Matura, el examen final de la enseñanza media de Austria, que da derecho a acceder a la enseñanza superior), conocimiento de idiomas (autopercepción del nivel) y estudios en curso de realización. El interés por obtener estos datos radica en la posibilidad de que alguna de estas variables tenga una incidencia en los estilos de aprendizaje, es decir, si existen diferencias en los estilos de aprendizaje, por ejemplo, entre hombres y mujeres, o entre los estudiantes que tienen el alemán como lengua materna y aquellos que no. Son estas variables independientes las que utilizamos para concretar la pregunta de la que partimos, a saber, si existen diferencias en los estilos de aprendizaje ligadas a alguna de estas. Tomamos como punto de partida una hipótesis nula, esto es, que no existe diferencia alguna en los estilos de aprendizaje ligada a las variables mencionadas; para comprobar o refutar esta hipótesis se utilizó la prueba t de Student.

\subsubsection{Diferencias significativas entre grupos}

En la gran mayoría de los casos, los resultados de la prueba t de Student confirman la hipótesis nula y apuntan a la inexistencia de diferencias basadas en la mayoría de las variables independientes tomadas en consideración. Sin embargo, se pudieron establecer diferencias significativas entre los siguientes grupos y ligadas a los siguientes factores:

- Por sexo en el estilo activo: se pudo constatar una diferencia significativa entre los resultados obtenidos por hombres y mujeres en el estilo activo, lo cual indica que de entre los estudiantes de español en la Universidad de Ciencias Económicas y Empresariales de Viena (Wirtschaftsuniversität Wien), los hombres son significativamente más activos que las mujeres en su 
aprendizaje. ${ }^{3}$ En el resto de estilos de aprendizaje no hay diferencias estadísticamente significativas entre los dos sexos, según se muestra en la Tabla 7.

Tabla 7. Distinción por sexo en las medias obtenidas en cada estilo de aprendizaje

\begin{tabular}{llccc}
\hline & Sexo & N & Media & S. D. \\
\hline estilo activo & hombres & 67 & 11,60 & 2,802 \\
\multirow{3}{*}{ estilo reflexivo } & mujeres & 131 & 10,27 & 3,595 \\
& hombres & 67 & 14,31 & 2,731 \\
estilo teórico & mujeres & 131 & 14,14 & 2,717 \\
& hombres & 67 & 13,34 & 2,643 \\
estilo pragmático & mujeres & 131 & 13,07 & 2,912 \\
& hombres & 67 & 12,75 & 3,125 \\
& mujeres & 131 & 12,18 & 3,168 \\
\hline
\end{tabular}

- Por sexo, en la autoevaluación del estilo pragmático: se pudo constatar una diferencia significativa entre la puntuación que se otorgan los hombres y las mujeres en el estilo pragmático, lo cual indica que los hombres se consideran más pragmáticos en su aprendizaje que las mujeres. ${ }^{4}$ En la Tabla 8 se puede comprobar, además, que en el resto de autoevaluaciones de los estilos de aprendizaje no hay diferencias significativas entre los dos sexos.

Tabla 8. Distinción por sexo en las medias obtenidas en la autoevaluación de los estilos de aprendizaje

\begin{tabular}{llccc}
\hline & Sexo & N & Media & S. D. \\
\hline autoevaluación & hombres & 67 & 13,81 & 3,893 \\
estilo activo & mujeres & 131 & 12,76 & 4,542 \\
autoevaluación & hombres & 67 & 14,43 & 3,714 \\
estilo reflexivo & mujeres & 131 & 13,99 & 5,451 \\
autoevaluación & hombres & 67 & 12,24 & 4,726 \\
estilo teórico & mujeres & 131 & 12,44 & 4,317 \\
autoevaluación & hombres & 67 & 12,88 & 4,284 \\
estilo pragmático & mujeres & 131 & 11,25 & 5,080 \\
\hline
\end{tabular}

3. Diferencia significativa entre los resultados obtenidos por hombres y mujeres en el estilo activo; prueba t de Student $\mathrm{t}(165)=2,85 ; \mathrm{p}=0,005$.

4. Diferencia significativa en la autoevaluación del estilo pragmático en las puntuaciones que se otorgan hombres y mujeres; prueba t de Student $\mathrm{t}(196)=2,25 ; \mathrm{p}=0,026$. 
- Por lengua materna alemán, respecto al estilo reflexivo. En este caso, hay una diferencia entre el grupo de estudiantes que tienen el alemán como lengua materna y aquellos que no lo tienen. Estos últimos tienen valores significativamente más altos en el estilo reflexivo. ${ }^{5}$ Esto indica que el grupo de estudiantes cuya lengua materna no es el alemán, pero están inmersos en el sistema educativo de Austria, donde esta es la principal lengua de comunicación, tienen valores más altos en el estilo reflexivo. Los valores obtenidos en cada uno de los estilos por ambos grupos se muestran en la Tabla 9.

Tabla 9. Diferencias en las medias obtenidas en cada estilo de aprendizaje basadas en lengua materna alemán o no

\begin{tabular}{llrcc}
\hline & Alemán LM & N & Media & S. D. \\
\hline estilo activo & sí & 169 & 10,63 & 3,373 \\
estilo reflexivo & no & 29 & 11,28 & 3,555 \\
\multirow{2}{*}{ estilo teórico } & sí & 169 & 14,00 & 2,736 \\
& no & 29 & 15,34 & 2,319 \\
estilo pragmático & sí & 169 & 13,20 & 2,832 \\
& no & 29 & 12,93 & 2,789 \\
& sí & 169 & 12,44 & 3,065 \\
& no & 29 & 11,93 & 3,683 \\
\hline
\end{tabular}

4-3.2 Factores que no implican diferencias en los estilos de aprendizaje Ya hemos mencionado arriba que respecto a la gran mayoría de variables independientes incluidas en el estudio no existen diferencias respecto a los estilos de aprendizaje, según indican las correspondientes pruebas $t$ de Student. Con la salvedad de las tres variables comentadas en el apartado anterior, los estudiantes muestran un perfil muy uniforme, puesto que no hay diferencias significativas en ninguno de los estilos de aprendizaje y tampoco en la autoevaluación respecto al resto de variables. Por motivos de espacio, resumimos a continuación los resultados, sin ofrecer las correspondientes pruebas estadísticas.

- No hay diferencias por nivel de estudios (estudiantes en programas de máster frente a otros en grados).

5. Los estudiantes que no tienen el alemán como lengua materna tienen valores significativamente más altos en el estilo reflexivo que aquellos que son hablantes nativos de alemán; prueba $\mathrm{t}$ de Student $\mathrm{t}(196)=-2,50 ; \mathrm{p}=0,013$. 
- No hay diferencias por provenir del sistema educativo austriaco o de otro distinto. Los estudiantes que han accedido a la universidad tras haber realizado el examen final de la enseñanza media austriaca, (Matura) no presentan valores distintos a aquellos que provienen de otros sistemas educativos ( $y$ han cursado previamente la enseñanza media en un país distinto).

- No hay diferencias entre aquellos participantes que dicen hablar más de 3 lenguas extranjeras y los que no.

Aparte de las variables anteriores, que no indican diferencias en los estilos de aprendizaje, hay algunos otros datos que simplemente no se han podido tener en cuenta por el reparto de la población estudiada. Por ejemplo, la edad de la gran mayoría de los participantes se sitúa entre los 20 y 23 años y fuera de esas edades el número de informantes es demasiado pequeño como para intentar establecer diferencias. Tampoco los datos respecto a la carrera elegida nos permiten interpretación alguna, puesto que la gran mayoría $(N=123)$ estudia Ciencias Empresariales Internacionales, y hay un grupo $(N=52)$ que estudia Ciencias Empresariales. La proporción del resto de carreras es demasiado pequeña para establecer una comparación y además, todas ellas comparten una orientación a las Ciencias Empresariales.

\subsection{Perfiles de aprendizaje}

Una vez analizados los datos para comprobar o refutar las hipótesis previas sobre las variables socioacadémicas, interesa establecer una visión general sobre los perfiles de aprendizaje concretos de la población estudiada. Es esta información, en definitiva, la que nos permitirá en un futuro programar y desarrollar nuestra actividad docente de un modo más acorde con la idiosincrasia particular de nuestros estudiantes. Para establecer los perfiles de aprendizaje, hemos utilizado el baremo general de preferencias en estilos de aprendizaje presentado en 4.1 (cf. Tabla 5) y hemos otorgado una puntuación entre 1 y 3 y cada uno de ellos según el criterio siguiente:

- Preferencia muy baja o baja: 1

- Preferencia moderada: 2

- Preferencia alta o muy alta: 3

$\mathrm{Al}$ respecto, cabe recordar que la posible puntuación en cada uno de los estilos de aprendizaje (de o a 20) no se divide uniformemente en simples tercios numéricos, sino que la valoración está ligada a la escala de valores de cada uno de los estilos (véase 4.1). 
La valoración de las preferencias en los distintos estilos de aprendizaje y su combinación nos permite establecer perfiles de aprendizaje. Una vez establecidos estos, es posible cuantificar los individuos que responden a un perfil determinado, lo que en una fase posterior contribuirá a poner en marcha intervenciones didácticas pertinentes para un mayor número de estudiantes.

A partir de los datos, hemos podido establecer que existen perfiles con preferencia alta o muy alta por alguno de los estilos de aprendizaje (y moderada o baja en los demás) y también perfiles de combinación, en los que existe una preferencia alta o muy alta en dos estilos. Los resultados concretos y la frecuencia de cada uno de los perfiles encontrados en los estudiantes de la Universidad de Ciencias Económicas y Empresariales de Viena (Wirtschaftsuniversität Wien) se pueden ver en la Tabla 10.

Tabla 10. Tipología de perfiles de aprendizaje de los estudiantes de español de la Universidad de Ciencias Económicas y Empresariales de Viena (Wirtschaftsuniversität Wien) (en orden de frecuencia)

\begin{tabular}{|c|c|c|c|}
\hline & $\mathbf{n}=$ & $\%$ & Descripción del perfil \\
\hline tipo 1 & 47 & $24 \%$ & $\begin{array}{l}\text { Ninguno de los estilos con preferencia alta o muy alta } \\
\text { (activo hasta menos de 13; reflexivo menos de 16; teórico menos de } 15 \text { y } \\
\text { pragmático menos de 15) }\end{array}$ \\
\hline tipo 2 & 28 & $14 \%$ & $\begin{array}{l}\text { Preferencia por el estilo pragmático } \\
(15 \text { o más puntos; en los demás, moderada o baja) }\end{array}$ \\
\hline tipo 3 & 23 & $12 \%$ & $\begin{array}{l}\text { Combinación reflexivo - teórico } \\
\text { (reflexivo } 16 \text { o más y teórico } 15 \text { o más; los otros, sin preferencia alta) }\end{array}$ \\
\hline tipo 4 & 21 & $11 \%$ & $\begin{array}{l}\text { Preferencia por el estilo teórico } \\
\text { (15 o más puntos; en los demás, moderada o baja) }\end{array}$ \\
\hline tipo 5 & 19 & $10 \%$ & $\begin{array}{l}\text { Preferencia por el estilo reflexivo } \\
\text { (16 o más puntos; en los demás, moderada o baja) }\end{array}$ \\
\hline tipo 6 & 19 & $10 \%$ & $\begin{array}{l}\text { Preferencia alta por tres o más estilos de aprendizaje } \\
\text { (solo } 1 \text { persona tiene preferencia alta por los } 4 \text { ) }\end{array}$ \\
\hline tipo 7 & 12 & $6 \%$ & $\begin{array}{l}\text { Combinación activo - pragmático } \\
\text { (activo } 13 \text { o más y pragmático } 15 \text { o más; los otros, sin preferencia alta) }\end{array}$ \\
\hline tipo 8 & 10 & $5 \%$ & $\begin{array}{l}\text { Preferencia por el estilo activo } \\
\text { (13 o más puntos; en los demás, moderada o baja) }\end{array}$ \\
\hline tipo 9 & 9 & $5 \%$ & $\begin{array}{l}\text { Combinación activo - reflexivo } \\
\text { (activo } 13 \text { o más y reflexivo } 16 \text { o más; los otros, sin preferencia alta) }\end{array}$ \\
\hline tipo 10 & 4 & $2 \%$ & $\begin{array}{l}\text { Combinación activo - teórico } \\
\text { (activo } 13 \text { o más y teórico } 15 \text { o más; los otros, sin preferencia alta) }\end{array}$ \\
\hline
\end{tabular}


Tabla 10. (continuado)

\begin{tabular}{|c|c|c|c|}
\hline & $\mathrm{n}=$ & $\%$ & Descripción del perfil \\
\hline tipo 11 & 4 & $2 \%$ & $\begin{array}{l}\text { Combinación teórico - pragmático } \\
\text { (teórico y pragmático } 15 \text { o más puntos; los otros, sin preferencia alta) }\end{array}$ \\
\hline tipo 12 & 2 & $1 \%$ & $\begin{array}{l}\text { Combinación reflexivo - pragmático } \\
\text { (reflexivo } 16 \text { o más y pragmático } 15 \text { o más; los otros, sin preferencia alta) }\end{array}$ \\
\hline
\end{tabular}

El primer dato significativo que nos proporciona la Tabla 10 es que el grupo más numeroso de estudiantes presenta un perfil en el que no hay una preferencia alta $o$ muy alta en ninguno de los estilos de aprendizaje. La lectura que se desprende de esto es que casi una cuarta parte de los estudiantes tiene amplias posibilidades de mejora. Sin embargo, la interpretación de estos datos no conlleva necesariamente una valoración negativa; en efecto, aunque no haya ninguna preferencia alta por alguno de los estilos, la presencia de índices moderados en cada uno de los estilos de aprendizaje implica posibilidades de cambio más reales que en otros casos y un equilibrio en el perfil cognitivo a partir del cual se puede construir cualquier mejora.

Otro grupo también muy numeroso es el que conforman los tipos 3,4 y 5 en conjunto: se trata de los perfiles con preferencia por el estilo reflexivo, por el estilo teórico o el perfil de combinación de estos dos estilos; un tercio de todos los estudiantes presenta uno de estos tres perfiles. Si abarcamos, pues, el primer tipo (con preferencias moderadas o bajas en todos los estilos de aprendizaje) y el grupo que acabamos de comentar establecemos que más de la mitad de la población analizada presenta posibilidades de mejora en los estilos pragmático y, sobre todo, activo.

Por cantidad de individuos, el tercer gran grupo (una cuarta parte del total) lo constituyen los tipos 2, 7 y 8: se trata esta vez de los perfiles con preferencia por el estilo pragmático, el perfil de combinación activo - pragmático y el perfil de preferencia por el estilo activo.

Por último, cabe destacar que un $10 \%$ del total de encuestados presenta un perfil con preferencia alta o muy alta en 3 o más estilos de aprendizaje. Solo una persona tiene preferencia alta en los cuatro estilos.

El resto de perfiles son muy minoritarios (con menos de 10 individuos por perfil) y corresponden a combinaciones que aparecían como no compatibles según la matriz de correlación de Pearson (cf. Tabla 6). A modo de resumen, presentamos en la Tabla 11 una síntesis de los grupos que conforman el alumnado analizado. 
Tabla 11. Resumen de los perfiles de aprendizaje de los estudiantes de español de la Universidad de Ciencias Económicas y Empresariales de Viena (Wirtschaftsuniversität Wien) (en orden de frecuencia)

\begin{tabular}{|c|c|c|}
\hline $\begin{array}{l}\text { Casos } \\
n=198\end{array}$ & $\%$ & Descripción del perfil \\
\hline 63 & $33 \%$ & $\begin{array}{l}\text { Preferencia por el estilo reflexivo, por el estilo teórico, o por la combinación } \\
\text { reflexivo-teórico }\end{array}$ \\
\hline 50 & $25 \%$ & $\begin{array}{l}\text { Preferencia por el estilo pragmático, por el estilo activo, o por la combinación } \\
\text { activo-pragmático }\end{array}$ \\
\hline 47 & $24 \%$ & Ninguno de los estilos con preferencia alta o muy alta \\
\hline 19 & $10 \%$ & Preferencia alta por tres o más estilos de aprendizaje \\
\hline 19 & $10 \%$ & $\begin{array}{l}\text { Otras combinaciones (activo-reflexivo, activo-teórico, teórico-pragmático y } \\
\text { reflexivo-pragmático) }\end{array}$ \\
\hline
\end{tabular}

\section{Discusión}

La primera constatación que podemos hacer respecto a los resultados presentados en el apartado anterior es que muestran una dicotomía clara entre las combinaciones reflexivo-teórico y activo-pragmático, y que constituyen una constelación muy distinta a la del alumnado descrito por otros estudios previos que hemos tomado como punto de referencia. La población universitaria española descrita por Alonso et al. (1994) presentaba mayoritariamente las correlaciones reflexivoteórico y teórico-pragmático, las mismas que el estudio realizado por Martínez Martínez (2004) con estudiantes japoneses. Los estudiantes de Económicas descritos por Alonso et al. (1994, p.138), sin embargo, presentaban en los estilos activo y pragmático valores medios más altos que los estudiantes universitarios del resto de carreras, lo que hasta cierto punto los acerca más a los estudiantes analizados en este trabajo. Asimismo, esto plantea la pregunta acerca de si un perfil de aprendizaje concreto (o algunas de características) pueden influir en la elección de unos estudios universitarios, en nuestro caso concreto, las Ciencias Empresariales.

Además, la lectura de estos datos nos lleva directamente a la reflexión de que metodologías didácticas que puedan tener resultados satisfactorios con cierto tipo de aprendientes, pueden ser menos efectivas, o menos apropiadas para ser utilizadas con otras poblaciones de estudiantes.

Respecto a los resultados concretos de los estudiantes de la Universidad de Ciencias Económicas y Empresariales de Viena (Wirtschaftsuniversität Wien) podemos afirmar lo siguiente: el grupo mayoritario de estudiantes (un tercio) pre- 
senta una preferencia alta bien en el estilo reflexivo, bien en el estilo teórico o en la combinación de ambos. En cuanto a la preferencia por el estilo reflexivo se tratará, pues, de estudiantes acostumbrados a recopilar y analizar datos, que prefieren intervenir solo cuando se sientan seguros para ello, por lo que tienden a observar y a prepararse con detenimiento. Los estudiantes con una preferencia alta por el estilo teórico comparten con los reflexivos la necesidad de sentirse seguros antes de participar abiertamente en clase, pues su perfeccionismo los llevará a intentar evitar errores. Estos estudiantes son lógicos y estructurados y querrán integrar sus conocimientos lingüísticos en teorías a partir de hipótesis previas. La preferencia por los estilos reflexivo y teórico o por su combinación parece concordar con el desarrollo de ciertas ventajas cognitivas que experimentan las personas bilingües o en proceso de formación bilingüe, en concreto la reflexividad sobre la lengua (Signoret, 2013). Sin embargo, las características de este grupo, que, como hemos dicho, es el más numeroso entre los estudiantes de nuestra institución, pueden también formularse de manera negativa, haciendo hincapié en las dificultades a las que se enfrentarán. Y es que estos estudiantes reflexivos y los teóricos mostrarán reticencias a la hora de llevar a la práctica los contenidos lingüísticos aprendidos, sobre todo si esta se realiza con una preparación previa que ellos consideran insuficiente. Habrá que llevarlos de manera gradual a probar cierto tipo de actividades que conllevan una actuación inmediata y mostrarles que estas experiencias, que a menudo ellos ven como obstáculos, son solo pequeños retos que los pueden ayudar a avanzar en su aprendizaje.

El segundo grupo más numeroso entre la población estudiada son los estudiantes con preferencia por el estilo pragmático, por el estilo activo, o por la combinación activo-pragmático. Si retomamos la caracterización que acabamos de hacer del grupo anterior, podemos afirmar que este grupo presenta unas preferencias totalmente opuestas al anterior. En efecto, los estudiantes con este perfil cognitivo no tendrán problemas si se trata de exponerse frente al resto de sus compañeros. Preferirán actividades en las que haya actuaciones inmediatas (p.ej. dramatizaciones o juegos de rol) y disfrutarán probando actividades nuevas y abiertas. Por el contrario, estos estudiantes darán menos importancia, o se aburrirán, si deben invertir mucho tiempo en las preparaciones previas de tareas o en las explicaciones exhaustivas de, por ejemplo, temas gramaticales.

Frente a estos dos perfiles cognitivos opuestos, la actitud del profesor deberá por tanto ser de precaución, siempre inclusiva y flexible a la hora de sugerir variaciones, sobre todo cuando lleve al aula cierto tipo de actividades que pueden ser vistas como obstáculos por parte de individuos con un estilo de aprendizaje concreto. No se trata en absoluto de no hacer uso de ellas, sino de darles la posibilidad de llevarlas a cabo de modo que todos los estudiantes se sientan cómodos. A modo de ejemplo, en el caso de preparar una simulación, para que los estudiantes 
reflexivos y teóricos pierdan el miedo a realizarla, sería posible permitir una preparación previa en casa y una posterior práctica en grupos pequeños en los que la exposición ante un público sea menor.

Teniendo en cuenta que el tercer grupo más numeroso entre la población estudiada no presenta una preferencia alta o muy alta en ninguno de los estilos de aprendizaje, cualquier intervención didáctica destinada a mejorar algún aspecto de alguno de los estilos debería ser beneficiosa para este grupo de aprendientes.

Por último, es necesario hacer referencia a las limitaciones de nuestro estudio, que se entrelazan con las investigaciones que es preciso llevar a cabo a partir de este análisis. En primer lugar, la necesidad de validar los resultados ante una muestra mayor de estudiantes del mismo perfil. A continuación, contrastar estos resultados con aquellos obtenidos en estudiantes de español en contextos específicos pertenecientes a otros ámbitos (Derecho, Sanidad o Turismo). Finalmente, conseguir diagnosticar los estilos de aprendizaje de los docentes para poder establecer las correlaciones oportunas y llegar, así, al verdadero diálogo entre enseñanza y aprendizaje que invocábamos al comienzo de la investigación.

\section{Conclusiones}

La didáctica de lenguas extranjeras, a lo largo de los años, ha ido evolucionando; la irrupción de cada nueva metodología de enseñanza normalmente ha significado en gran parte el rechazo a metodologías anteriores. Al poner de manifiesto todas las posibles (o percibidas) debilidades de métodos previos, frente a las posibles (o percibidas) ventajas de enfoques más novedosos, las aulas de idiomas a menudo se convierten en entornos en los que no hay lugar para maneras de aprender diversas.

La cantidad de teorías e investigaciones sobre los estilos de aprendizaje pone de manifiesto el atractivo de considerar los diferentes modos de aprender como un factor esencial en la enseñanza (Dörnyei y Skehan, 2003; Griffiths, 2012), que, a pesar de todas las críticas reseñadas en apartados anteriores, parece estar consolidado incluso desde un punto de vista institucional, puesto que tanto el Marco común europeo de referencia para las lenguas (Consejo de Europa, 2002) como el Plan curricular del Instituto Cervantes (2006) los incluyen como una variable en el proceso de enseñanza y aprendizaje de lenguas.

Esta situación contrasta, sin embargo, con la baja incidencia real que probablemente tenga la diferenciación de estilos en el aula, puesto que ni siquiera su diagnóstico parece estar presente en la enseñanza de ELE (Domínguez Pelegrín et al., 2019, p. 439). La idea de que los profesores enseñan usando el modo en que ellos aprendieron mejor (Kinsella, 1995; Dörnyei, 2005, p.156) apunta a la exis- 
tencia de ciertas preferencias a la hora de aprender que, quizá inconscientemente, consideremos más apropiadas, más eficientes o en el peor de los casos, las únicas válidas. Es por ello que una primera recomendación práctica debería consistir en concienciar al profesorado de la existencia de diferencias entre los estudiantes, para que estas se vean no como una amenaza, sino como una oportunidad.

En concreto, este estudio ha revelado claramente la existencia de tres grupos distintos de estudiantes en nuestra institución: uno más bien neutro (ningún estilo desarrollado) y dos opuestos (combinación activo-pragmático frente a combinación reflexivo-teórico). Obviamente, los tres grupos tienen el mismo derecho y la misma necesidad de recibir el input didáctico que para ellos es el más adecuado y el profesorado debe intentar ofrecer a todos ellos las mejores condiciones para su aprendizaje y, asimismo, intentar que aprovechen nuevos modos de aprender.

En este sentido, el diagnóstico del perfil de aprendizaje mediante el uso de un cuestionario apropiado tiene ya de por sí un efecto colateral importante previo a cualquier intervención didáctica: el tratamiento directo del tema en el aula lleva a los estudiantes a reflexionar sobre sus estilos de aprendizaje y de esta manera es posible dar cuenta de la existencia de otras formas de aprender que también se pueden desarrollar y eventualmente, comenzar a usar (Ehrman, 1996; Cohen, 2002).

La opción pedagógica más relevante será, por tanto, conseguir la autoconsciencia y el metaconocimiento de los estudiantes acerca de su perfil cognitivo y de su estilo en el aprender. El proceso los llevará, sin duda, a promocionar sus competencias, entre ellas la de la autonomía, en sintonía con los procesos reclamados para el nuevo Espacio Europeo de Educación Superior a partir de la Declaración de Bolonia y otros documentos europeos (Comisión Europea, 2008, p.11). Es en este marco donde consideramos que la autorregulación de los estilos de aprendizaje constituye una adecuada vía para aspirar a dicha autonomía.

Los docentes, independientemente de las creencias que tengan respecto al aprendizaje de lenguas extranjeras, deben adoptar una actitud ecléctica, ser conscientes de las diferencias individuales de los aprendientes, dar cabida en el aula a las distintas maneras de aprender y facilitar una mejora en los perfiles de aprendizaje. Además, también deben orientar su manera de enseñar al perfil dominante entre sus estudiantes y suministrarles el input que necesitan. La realización de un diagnóstico y análisis de los estilos de aprendizaje del alumnado permite a los docentes tomar decisiones metodológicas adaptadas y es un primer paso en esta dirección. 


\section{Información sobre financiación}

Agradecemos a la Wirtschaftsuniversität Wien la financiación de esta investigación mediante un Scholarship of Teaching and Learning (2018).

\section{Referencias}

Acevedo-Pierart, C. G. (2011). Learning styles, gender and academic performance. Journal of Learning Styles, 8(4), 71-84.

Aguilar, M.C. (2010). Learning styles and learning strategies in university students. Revista de Psicología, 28(2), 207-226.

Alducin-Ochoa, J.M., y Vázquez-Martínez, A. I. (2017). Learning styles, socio-demographic variables and academic performance of building Engineering Students. Educare Electronic Journal, 21(1), 1-31.

Allinson, C., y Hayes, J. (1996). The cognitive style index: A measure of intuition-analysis for organizational research. Journal of Management Studies, 33(1), 119-136. https://doi.org/10.1111/j.1467-6486.1996.tboo801.x

Allueva-Torres, P., y Bueno-García, C. (2011). Learning styles and thinking styles of university students. Learning to learn and learning to think. ARBOR Ciencia, Pensamiento y Cultura, 187(3), 261-266. https://doi.org/10.3989/arbor.2011.Extra-3n3155

Almigbal, T.H. (2015). Relationship between learning styles preferences of medical students and academic achievement. Saudi Medical Journal, 36(3), 349-355.

https://doi.org/10.15537/smj.2015.3.10320

Alonso, C. M., Gallego, D. J., y Honey, P. (1994). Los estilos de aprendizaje. Procedimientos de Diagnóstico y Mejora. Bilbao: Editorial Mensajero.

Álvarez-Montero, F. J., Leyva-Cruz, M.G., y Moreno-Alcaraz, F. (2018). Inventarios de estilos de aprendizaje: una actualización de la matriz de fiabilidad y validez de Coffield, Moseley, Hall, y Ecclestone. Electronic Journal of Research in Educational Psychology, 16(46). https://doi.org/10.25115/ejrep.v16i46.2237

An, D., y Carr, M. (2017). Learning styles theory fails to explain learning and achievement: Recommendations for alternative approaches. Personality and Individual Differences, 116, 410-416. https://doi.org/10.1016/j.paid.2017.04.050

Bangcola, A.A. (2016). Learning styles as predictor of academic performance in the Nursing Department of an Asian University and colleges" International Journal of Learning, Teaching and Educational Research, 15(4), 22-31.

Coffield, F., Moseley, D., Hall, A., y Ecclestone, K. (2004). Learning Styles and Pedagogy in Post-16 Learning: A Systematic and Critical Review. London: Learning and Skills Research Centre.

Cohen, A.D. (2002). Preparing teachers for styles- and strategies-based instruction. En V. Crew, C. Davison y B. Mak (Eds.), Reflecting on language in education. (pp. 49-69). Hong Kong: The Hong Kong Institute of Education.

Cohen, A.D. (2007). Coming to terms with language learner strategies: Surveying the experts. En A. Cohen y E. Macaro (Eds.) Language learner strategies (pp. 29-46). Oxford: Oxford University Press. 
Cohen, A. D., Oxford, R. L., y Chi, J.C. (2001). Learning Style Survey. Recuperado el 4 de diciembre de 2019 de https://carla.umn.edu/maxsa/documents/LearningStyleSurvey _MAXSA.pdf

Comisión Europea (2008). El Marco Europeo de Cualificaciones para el aprendizaje permanente (EQF-MEC). Luxemburgo: Oficina de Publicaciones Oficiales de las Comunidades Europeas.

Consejo de Europa (2002). Marco común europeo de referencia para las lenguas: aprendizaje, enseñanza, evaluación. Madrid: MECD y Grupo Anaya.

Domínguez Pelegrín, J., López Quero, S., y Andión Herrero, M.A. (2019). Las creencias de los profesores de español como lengua extranjera sobre los estilos de aprendizaje. Revista Española de Lingüistica Aplicada, 32(2), 419-454. https://doi.org/10.1075/resla.17041.dom

Dörnyei, Z. (2005). The psychology of the language learner: Individual differences in second language acquisition. (Second language acquisition research). Mahwah, N.J.: L. Erlbaum.

Dörnyei, Z., y Ryan, S. (2015). The psychology of the language learner revisited (Second language acquisition research series). New York, NY: Routledge/Taylor y Francis Group. https://doi.org/10.4324/9781315779553

Dörnyei, Z., y Skehan, P. (2003). Individual differences in second language learning. En C. J. Doughty y M.H. Long (Eds.), The Handbook of Second Language Acquisition (pp. 589-630). Oxford: Blackwell. https://doi.org/10.1002/9780470756492.ch18

Dunn, R., y Dunn, K. (1978). Teaching students through their individual learning styles: a practical approach. New Jersey: Prentice Hall.

Dunn, K., Dunn, R., y Price, G.E. (1989). Learning style inventory. Lawrence, KS: Price Systems.

Ehrman, M.E. (1996). Understanding second language difficulties. Thousand Oaks, CA: Sage.

Ehrman, M., y Leaver, B.L. (2003). Cognitive styles in the service of language learning. System, 31(3), 393-415. https://doi.org/10.1016/S0346-251X(03)00050-2

Ellis, R. (1994). The study of Second Language Acquisition. Oxford: Oxford University Press.

Ellis, R. (2004). Individual Differences in Second Language Learning. En A. Davies y C. Elder (Eds.), The Handbook of Applied Linguistics (pp. 525-551). Oxford: Blackwell. https://doi.org/10.1002/9780470757000.ch21

Escurra Mayaute, L.M. (2011). Análisis psicométrico del Cuestionario de Honey y Alonso de Estilos de Aprendizaje (CHAEA) con los modelos de la Teoría Clásica de los Test y de Rasch, Persona, 14, 71-109. https://www.redalyc.org/articulo.oa?id=147122650003

Felder, R.M., y Silverman, L. K. (1988). Learning Styles and Teaching Styles in Engineering Education. Engineering Education, 78, 674-681.

Flores-Mejía, J.G., Velázquez-Gatica, B., y Gaona-Rojas, F. (2014). Actitudes, estrategias y estilos de aprendizaje en estudiantes universitarios de Psicología. XI Coloquio Internacional Multidisciplinario. Unidad Profesional del Balsas de la Universidad Michoacana de San Nicolás de Hidalgo (pp. 1-14). Huetamo, Michoacán. Recuperado de http://www.guillermofloresuagro.net/uploads/5/8/4/5/58453193/actitudes_estrategias_y _estilos_de_aprendizaje_en_estudiantes_universitarios_de_psicologi\%CC\%81a.pdf

Freiberg Hoffmann, A., D’Anna, A., Vigh, C., y Berenguer, D. (2017). Estilos de aprendizaje y su relación con variables sociodemográficas y académicas en estudiantes universitarios de Buenos Aires. ACADEMO. Revista de Investigación en Ciencias Sociales y Humanidades, 4(1). Recuperado de https://revistacientifica.uamericana.edu.py/index.php/academo /article/view/67

Gregersen, T., y MacIntyre, P.D. (2014). Capitalizing on language learner individuality. From premise to practice. Bristol: Multilingual Matters. https://doi.org/10.21832/9781783091218 
Griffiths, C. (2012). Learning Styles: Traversing the Quagmire. En S. Mercer, S. Ryan y M. Williams (Eds), Psychology for Language Learning. Insights from theory, research and practice (pp. 151-168). London: Palgrave Macmillan.

https://doi.org/10.1057/9781137032829_11

Honey, P., y Mumford, A. (1986). Using your learning styles. Maidenhead: Peter Honey.

Honey, P., y Mumford, A. (1986b). Learning Styles Questionnaire. London: Peter Honey Publications Ltd.

Honey, P., y Mumford, A. (1994). The Manual of Learning Styles. Revised version Maidenhead: Peter Honey.

Instituto Cervantes (2006). Plan curricular del Instituto Cervantes: niveles de referencia para el español. Madrid: Instituto Cervantes, Biblioteca Nueva y Editorial Edelsa.

Jahanbakhsh, R. (2012). Learning styles and academic achievement: a case study of iranian high school girl's students. Procedia. Social and Behavioral Sciences, 51, 1030-1034. https://doi.org/10.1016/j.sbspro.2012.08.282

Keefe, J.W. (Ed.). (1979). Student learning styles: diagnosing and prescribing programs. Reston: National Association of Secondary School Principals.

Kinsella, K. (1995). Understanding and empowering diverse learners in ESL classrooms. En J. Reid (Ed.), Learning styles in the ESL/EFL classroom (pp.170-194). Boston: Heinle \& Heinle.

Kirschner, P.A. (2017). Stop propagating the learning styles myth. Computers \& Education, 106, 166-171. https://doi.org/10.1016/j.compedu.2016.12.006

Kirschner, P.A., y van Merriënboer, J. J.G. (2013). Do Learners Really Know Best? Urban Legends in Education, Educational Psychologist, 48(3), 169-183. https://doi.org/10.1080/00461520.2013.804395

Kolb, D.A. (1976). Learning Style Inventory: Technical Manual. Boston: McBer \& Company (1984, revised edition).

MacIntyre, P.D., Gregersen, T., y Clément, R. (2016). Individual Differences. En G. Hall (Ed.), The Routledge Handbook of English Language Teaching (pp. 310-323) London / New York: Routledge. https://doi.org/10.4324/9781315676203-27

Martínez Martínez, I. (2004). Nuevas perspectivas en la enseñanza-aprendizaje de ELE para japoneses: la concienciación formal. Tesis doctoral. Madrid: Universidad Complutense de Madrid. Recuperada el 03/o9/2019 de http://eprints.ucm.es/4338/

Oxford, R. (1993). Style Analysis Survey (SAS). Tuscaloosa: University of Alabama.

Oxford, R. L. (1999). 'Style wars' as a source of anxiety in language classrooms. En D. J. Young (Ed.), Affect in foreign language and second language learning (pp. 216-237). Boston: McGraw-Hill.

Pashler, H., McDaniel, M., Rohrer, D., y Bjork, R. (2008). Learning styles: Concepts and evidence. Psychological Science in the Public Interest, 9(3), 103-119. https://doi.org/10.1111/j.1539-6053.2009.01038.x

Peacock, M. (2001). Match or mismatch? Learning styles and teaching styles in EFL. International Journal of Applied Linguistics, 11, 1-20. https://doi.org/10.1111/1473-4192.00001

Pinilla-Herrera, A., y Cohen, A.D. (2019). Estilos y estrategias de aprendizaje. En J. Muñoz-Basols, E. Gironzetti y M. Lacorte (Eds.), The Routledge Handbook of Spanish Language Teaching (pp. 38-51). London / New York: Routledge.

Reid, J.M. (Ed.) (1995). Learning styles in the ESL/EFL classroom. Boston: Heinle and Heinle. Renés, P., y Martínez Geijo, P. (2015). Estilos de enseñanza y aprendizaje. Bilbao: Mensajero. 
Signoret Dorcasberro, A. (2013). Bilingüismo en la infancia. México: Centro de Enseñanza de Lenguas Extranjeras-UNAM.

Tait, H., y Entwistle, N. J. (1996). Identifying students at risk through ineffective study strategies. Higher Education, 31, 97-116. https://doi.org/10.1007/BFo0129109

Tomlison, C.A. (2014). The Differentiated Classroom: Responding to the Needs of all Learners. (2nd edition). Alexandria, VA: ASCD.

Van Zwanenberg, N., Wilkinson, L. J., y Anderson, A. (2000). Felder and Silverman's Index of Learning Styles and Honey and Mumford's Learning Styles Questionnaire: How do they compare and do they predict academic performance? Educational Psychology, 2o(3), 365-38o. https://doi.org/10.1080/713663743

Ventura, A.C., y Moscoloni, N. (2015). Learning styles and disciplinary differences: A crosssectional study of undergraduate students. International Journal of Learning and Teaching, 1(2), 88-93. https://doi.org/10.18178/ijlt.1.2.88-93

Vermunt, J. D. (1994). Inventory of Learning Styles (ILS) in higher education: scoring key. Tilburg. The Netherlands: Tilburg University, Department of Educational Psychology.

Villamizar-Acevedo, G. (2012). Learning styles and academic performance of students in Civil Engineering. Informes Psicológicos, 12(2), 41-54.

Yates, G.C.R. (200o). Applying learning style research in the classroom: Some cautions and the way ahead. En R.J. Riding y S. G. Rayner (Eds.), Interpersonal perspectives on individual differences (Vol. I: Cognitive styles, pp. 347-364). Stamford: Ablex.

Young, D. J. (1991). Creating a low-anxiety classroom environment: What does language anxiety research suggest? Modern Language Journal, 75, 426-439. https://doi.org/10.1111/j.1540-4781.1991.tbo5378.x

\title{
How Business Students Learn Spanish: Learning styles and didactic implications
}

\begin{abstract}
Students at WU (Vienna University of Economics and Business) may opt to learn Spanish as part of their degree program. As a first step towards making those who do so more aware of their preferred learning strategies, and thus promote autonomous learning, a study of their learning profiles was conducted. For this purpose, a German translation of the CHAEA questionnaire (Alonso et al., 1994) was produced and a digital survey designed. Both were piloted and later reviewed by a panel of five experts. The survey was sent to 744 students, of whom 198 responded (response rate: $26.6 \%$ ), providing a highly significant sample for the study. The responses indicate that business students predominantly display one of two opposing learning profiles: while some combine reflective and theoretical styles, others display active and pragmatic ones. This dichotomy, which contrasts with the results obtained from other learner populations, suggests that business students are in a position to benefit from any learning situation, since they possess strategies drawn from all learning styles.
\end{abstract}

Keywords: learning styles, cognitivism, Spanish as a foreign language, learning autonomy, individual differences, Spanish for specific purposes 


\section{Dirección para la correspondencia}

Pilar Pérez Cañizares

Department of Foreign Language Business Communication

Vienna University of Economics and Business

Welthandelsplatz 1

1020 Wien

Austria

pperezca@wu.ac.at

(iD) https://orcid.org/oooo-0oo2-9577-699X

\section{Co-author information}

Inmaculada Martínez Martínez

Centro Internacional de Estudios Superiores del Español - Comillas

martinezi@fundacioncomillas.es

D https://orcid.org/oooo-0003-4760-0903
Johannes Schnitzer

Institut für Romanische Sprachen

Wirschaftsuniversität Wien

johannes.schnitzer@wu.ac.at

(D) https://orcid.org/oooo-0oo2-9087-7134

\section{Historia de la publicación}

Date received: 16 December 2019

Date accepted: 3 December 2020 